TURIZAM

Volume 25, Issue 1

31-44 (2021)

ORIGINAL

SCIENTIFIC PAPER

\title{
Index of Destination Attractiveness: a Quantitative Approach for Measuring Tourism Attractiveness
}

\author{
Naser Ul Islam ${ }^{\mathrm{A}^{*}}$, Manjula Chaudhary ${ }^{\mathrm{A}}$ \\ Received: Jun 2020 | Accepted: November 2020 \\ DOI: $10.5937 /$ turizam25-27235
}

\begin{abstract}
The common perceptions about tourist destinations often hold even in the absence of facts and evidences. This research is an attempt to analyze the ground behind generic perceptions about tourism attractiveness of Kashmir valley. This has been done through primary survey of most important stakeholders; the visiting tourists. The data collected from 370 tourists has been used to deconstruct tourism attractiveness into different parameters and an Index of Destination Attractiveness has been prepared to understand the importance of each parameter to overall attractiveness. The evidences support the common perception that natural attractions play very important role in tourism attractiveness of valley but valley lacks other tourism motivators and falls short on most of the hygiene factors. These findings can be used to increase the attractiveness of valley by working on weak areas and the Index developed for the study can be used as a standard tool for continuous monitoring of attractiveness.
\end{abstract}

Keywords: Kashmir valley, Tourism attractiveness, Tourists' perception, Index of Destination Attractiveness, Motivation and Hygiene in tourism

\section{Introduction}

Tourism attractiveness of a destination is often considered to be one of the key determinants of its tourism pull. It reflects feelings, opinions, and perceptions of tourists about the destination's perceived ability to satisfy a vacation need (Hu, Ritchie, 1993; Mayo, Jarvis, 1981). Attractiveness displays the special features of a destination that makes it attractive to tourists (Cho, 2008). While, researchers found it dependent on the availability of resources and perceived values of these resources (Formica, Uysal, 2006). The concept relates to the destination image and influences the destination image (Fakeye, Crompton, 1991; Krešić, Prebežac, 2011; Zhou, 2005). Tourism attractiveness stems from the natural and man-made features. Researchers identified primary and secondary features behind the attractiveness of the destination (Morachat, 2003; Laws, 1995). Nature, culture, and traditional architecture were considered primary features and tourism infrastructure such as accommodation, transport, tourist services and facilities

A Department of Tourism and Hotel Management, Kurukshetra University Kurukshetra Haryana, India (136119); Corresponding author: islamnasiro8o@gmail.com 
as secondary features. Various studies drew an analogy from Herzberg's theory of motivation and equated primary features into motivating factors and secondary features into hygiene factors and both were considered equally important for attractiveness of destination (Crompton, 2003; Jensen, 2007; Tkaczynski, Thiele, 2013).

The existing studies on tourism destination attractiveness posit the need for a consistent enquiry into the evolving measures of tourism attractiveness, forces behind these measures, and getting a workable measure with practical application. This research paper is an attempt to conduct an enquiry on these lines in the context of Kashmir valley in India which is a popular tourist place in north India and its natural beauty is commonly perceived to that of Switzerland. The reality of this common perception and its association with tourism attractiveness has not been studied and this study is an attempt to fill this gap by developing an Index of Destination Attractiveness (IDA) that can evolve and be used regularly. The main research query was addressed through the following research objectives;

1. Identification of the factors of tourism attractiveness of Kashmir valley

2. Finding the attractiveness level of the Kashmir valley on the identified factors

3. Designing a tourism attractiveness index of Kashmir valley

4. To get insight into the possible course of actions for improving the attractiveness of different factors and overall attractiveness

\section{Study site description}

Kashmir valley a popular tourist attraction in northern India Himalayas having boundaries with China and Pakistan. Sixteenth century European traveler Bernier described "Kashmir paradise of Indies" (Lawrence, 1895). The Government of India crowned it as "Switzerland of India" (Chaudhary, 2010). The valley holds rich natural and cultural resources and the main attractions are mountains, snow, gardens, pastures, wildlife, water bodies, handicrafts, and religion (Bhat, Shyju, 2014; Chaudhary, Islam, 2020; Ganie, Dar, 2020). The location and natural beauty of Kashmir have made it a world-famous tourist destination and has a huge market for niche tourism (IBEF, 2017).

Kashmir valley has undergone tremendous changes since the $16^{\text {th }}$ century in terms of its social, cultural, political, and religious environment. The destination choice set of Indian and foreign tourists has expanded due to easy access to global destinations and these mobile and experienced tourists perceive a destination on a relative scale creating a need for updated narrative on the attractiveness of Kashmir valley in today's context.

\section{Literature Review}

The available literature on tourism attractiveness has theoretical and managerial connotations. It deals with the abstract, fluid, and ever-evolving concept and constructs of attractiveness. The role of attractiveness in tourism has been researched with a focus on quantification of measurement of attractiveness for practical purposes.

The concept of tourism attractiveness is abstract in nature though its measurement assists destination managers in comparing destinations and their competitiveness (Dupeyras, MacCallum, 2013; Gearing et. al., 1974). Tourism attractiveness measurement facilitates in identifying and strengthening the poor elements of destinations (Bhat, Malik, 2015; Chaudhary et al., 2017; Edward, George, 2008; Kumar, Dar 2017). 
Attractiveness in tourism is multidimensional. Earlier divided into five dimensions namely natural, social, historical, recreational, and infrastructural features (Gearing et al., 1974). These were later extended to seven dimensions with the addition of price levels and visitor's satisfaction (Dupeyras, MacCallum, 2013; Ritchie, Zins, 1978). Three methods have been adopted to study these constructs; demand side (Blazeska et al., 2015; Hu, Ritchie, 1993; Kim, 1998; Morachat, 2003; Pompurová, Šimočková, 2014; Reitsamer et al., 2016; Vengesayi et al., 2009) supply-side (Kaur, 1981; Smith, 1987) and mix method (Castro et al., 2015; Edward, George, 2008; Formica, Uysal, 2006; Paul, 2017). However, the demand-side method is considered the most effective and used method to judge the quality of attractiveness (Blazeska et al., 2015).

The use of multiple constructs to measure destination attractiveness led to the development of a single Index of Destination Attractiveness (IDA). The purpose of IDA is to merge several interrelated measures into a single measure (Smith, 1987). Krešić, Prebezac (2011) proposed an index for measuring tourism attractiveness.

IDA helps in the quantification of attractiveness by measuring the attractiveness of each attraction individually and then merging these values into aggregate values (Krešić, Prebezac, 2011). The designing of an attractiveness index model requires identification of indicators that motivate tourists (Cugno et al., 2012). Identification of touristic pull factors and converting them into measurable units was found difficult (Mukhopadhyay, 2011). Indexing attractiveness has received adequate attention in academic research but it is yet to be used as a managerial technique to consistently track and improve destinations.

The review of literature on destination attractiveness leads to the following inferences;

1. Tourism attractiveness is an evolving measure

2. Factors of tourism attractiveness are destination specific and change with time

3. Tourism attractiveness can depend upon the experiences and attitudes of tourists

4. Tourism attractiveness can be built based on consistent inputs on different factors of attractiveness from different stakeholders

5. The tools and methods of measurement of tourism attractiveness require are not varied and non-comparable.

The above inferences form the basis of enquiry for this paper and an attempt has been made to find the gaps between the generic perceptions about tourism attractiveness of Kashmir valley and perceptions of tourists visiting Kashmir valley and this in situ study to develop a measure of attractiveness. Kashmir valley is commonly perceived parallel in natural beauty to Switzerland however no study is available to link this perceived beauty to tourism attractiveness. This makes the concept of tourism attractiveness an interesting subject of study for the valley.

\section{Methodology}

The tourism attractiveness of Kashmir valley has been assessed based on perceptions of visiting tourists during their stay in Kashmir. The perceptions have been captured through primary data collected with the help of the questionnaire on the selected parameters. These parameters were selected considering these as influencing factors for attractiveness based on past studies Castrol et al. (2015), Das et al. (2007), Krešić, Prebežac, (2011), Kim (1998), Morachat (2003), Sharma (2016) and discussion with tourism experts. In all 39 parameters were chosen and responses of tourists were collected on a five-point Likert type scale using endpoint descriptions of very low (1) to very high (5). The questionnaire was pretested on 50 tourists in the summer of 2018 and 
was validated. The final questionnaire was used to collect data in two seasons; winters of 2018 and summers of 2019 to avoid the impact of seasonality and other events on tourists' perceptions. The respondents were approached at popular tourist sites of Srinagar, Gulmarg, Pahalgam, and Sonmarg. Tourists were also approached at Srinagar international airport. Each respondent was approached personally by the researcher and was briefed on the importance of study and the value of feedback before getting the questionnaire filled to ensure effective answers. In all 400 questionnaires were got filled out of which 370 (92.5\%) were found usable.

\section{Results}

\section{Descriptive analysis}

Descriptive analysis has been used to know the demographic profile of 370 tourists and is presented in Table 1.

Table 1. Tourists profile

\begin{tabular}{|c|c|c|c|}
\hline \multicolumn{2}{|c|}{ Variables } & Number & Percent \\
\hline \multirow{4}{*}{ Gender } & Male & 205 & 55.4 \\
\cline { 2 - 4 } & Female & 165 & 44.6 \\
\hline \multirow{4}{*}{ Age } & Below 20 & 32 & 8.6 \\
\cline { 2 - 4 } & $21-35$ & 199 & 53.8 \\
\cline { 2 - 4 } & $36-50$ & 98 & 26.5 \\
\cline { 2 - 4 } & 51-65 & 33 & 8.9 \\
\cline { 2 - 4 } & above 65 & 8 & 2.2 \\
\hline \multirow{4}{*}{ Marital status } & Married & 218 & 58.9 \\
\cline { 2 - 4 } & Unmarried & 152 & 49.1 \\
\hline \multirow{4}{*}{ Qualification } & Below graduate & 53 & 14.3 \\
\cline { 2 - 4 } & Graduate & 164 & 44.3 \\
\cline { 2 - 4 } & Post graduate & 142 & 38.4 \\
\cline { 2 - 4 } & Other & 11 & 3.0 \\
\hline \multirow{4}{*}{ Nationality } & Indian & 201 & 54.3 \\
\cline { 2 - 4 } & Foreign & 169 & 45.7 \\
\hline
\end{tabular}

The demographic analysis of tourists showed male (55.4\%), married (58.9\%), graduates (44.3\%), age group of $21-35$ (53.8\%) tourists in the majority. The domestic tourists were 54.3 percent while international tourists represented 45.7 percent.

\section{Exploratory factor analysis}

The data on different factors of attractiveness was tested before further analysis. Exploratory factor analysis (EFA) was run to extract the dimensionality of tourism attractiveness. The results of this step showed KMO of 0.865 and significant Bartlett's test of sphericity at 0.001. The KMO above recommended level indicates that data is appropriate for further analysis (Kothari, Garg, 2014; Malhotra, Briks, 2006). 
Table 2. EFA results

\begin{tabular}{|c|c|c|c|c|c|c|c|c|c|c|c|}
\hline Variables & FAC1 & FAC2 & $\mathrm{FAC3}$ & FAC4 & FAC5 & FAC6 & FAC7 & FAC8 & FAC9 & FAC10 & FAC11 \\
\hline X1-Transportation cleanliness & 0.798 & 0.079 & 0.258 & 0.050 & 0.044 & 0.072 & -0.046 & 0.124 & 0.118 & 0.031 & 0.029 \\
\hline X2-Sites cleanliness & 0.783 & 0.019 & 0.180 & 0.092 & 0.127 & 0.091 & -0.085 & 0.077 & 0.077 & 0.052 & 0.087 \\
\hline X3-food cleanliness & 0.737 & 0.169 & 0.163 & 0.084 & 0.037 & 0.215 & -0.005 & 0.000 & 0.074 & -0.025 & 0.023 \\
\hline X4-Accommdaion cleanliness & 0.703 & 0.170 & 0.079 & 0.331 & 0.120 & 0.152 & -0.047 & -0.012 & -0.025 & 0.046 & -0.096 \\
\hline X5-F\&B quality & 0.119 & 0.811 & 0.118 & 0.117 & 0.128 & 0.082 & -0.015 & 0.059 & 0.026 & 0.021 & 0.051 \\
\hline X6-F\&B varieties & 0.078 & 0.752 & 0.201 & 0.218 & 0.111 & 0.194 & -0.124 & 0.041 & 0.034 & 0.022 & 0.028 \\
\hline X7-Local food & 0.062 & 0.737 & 0.018 & 0.023 & 0.021 & 0.062 & -0.023 & 0.328 & 0.087 & -0.009 & 0.047 \\
\hline X8-customer support & 0.205 & 0.611 & 0.231 & 0.249 & 0.124 & 0.089 & 0.047 & 0.135 & 0.163 & 0.105 & -0.023 \\
\hline X9-Hospitality & 0.099 & 0.433 & 0.114 & 0.285 & 0.079 & 0.183 & 0.112 & 0.196 & 0.114 & 0.095 & -0.023 \\
\hline X10-Inside transport & 0.217 & 0.118 & 0.814 & 0.145 & 0.072 & 0.155 & 0.005 & 0.087 & 0.076 & 0.072 & 0.066 \\
\hline X11-Accessibility over world & 0.239 & 0.101 & 0.706 & 0.086 & 0.043 & 0.206 & 0.058 & 0.141 & 0.104 & .0 .000 & 0.013 \\
\hline X12-Transport quality & 0.250 & 0.217 & 0.701 & 0.058 & 0.041 & 0.108 & 0.115 & 0.047 & 0.166 & 0.197 & 0.042 \\
\hline X13-Road signage's & 0.273 & 0.179 & 0.646 & 0.020 & 0.130 & 0.040 & 0.267 & 0.001 & 0.142 & 0.070 & 0.036 \\
\hline X14-Shopping & 0.177 & 0.024 & 0.443 & 0.388 & 0.219 & 0.160 & 0.112 & 0.217 & 0.019 & 0.085 & 0.186 \\
\hline X15-Accommdation varieties & 0.130 & 0.180 & 0.120 & 0.787 & 0.060 & 0.058 & 0.060 & 0.090 & 0.057 & 0.216 & 0.004 \\
\hline 16-Accommodation quality & 0.283 & 0.274 & 0.067 & 0.738 & 0.053 & 0.090 & 0.117 & -0.002 & 0.039 & 0.036 & 0.002 \\
\hline X17Accommodation location & 0.180 & 0.134 & 0.074 & 0.735 & 0.123 & 0.052 & 0.025 & 0.204 & 0.043 & 0.131 & 0.061 \\
\hline X18-Recreational activities & 0.055 & 0.158 & 0.387 & 0.404 & 0.276 & 0.121 & 0.045 & 0.114 & 0.290 & 0.141 & 0.111 \\
\hline X19-Pahalgam & 0.039 & 0.043 & 0.112 & 0.063 & 0.833 & -0.004 & 0.025 & 0.014 & 0.095 & 0.080 & -0.002 \\
\hline X20-Sonmarg & 0.086 & 0.051 & 0.048 & 0.115 & 0.800 & 0.052 & -0.025 & 0.073 & -0.010 & 0.058 & 0.123 \\
\hline X21-Gulmarg & 0.044 & 0.204 & 0.169 & 0.004 & 0.777 & 0.039 & -0.064 & -0.075 & 0.036 & 0.169 & -0.087 \\
\hline X22-Srinagar & 0.138 & 0.185 & 0.041 & 0.095 & 0.611 & 0.140 & -0.071 & 0.106 & 0.030 & 0.079 & 0.102 \\
\hline X23-Cellular services & 0.130 & 0.091 & 0.142 & 0.065 & 0.041 & 0.843 & -0.118 & -0.029 & 0.135 & -0.017 & 0.049 \\
\hline X24-Internet services & 0.080 & 0.212 & 0.127 & 0.013 & 0.094 & 0.782 & 0.150 & 0.031 & 0.243 & 0.059 & 0.003 \\
\hline X25-Banking- ATM & 0.302 & 0.138 & 0.174 & 0.118 & 0.099 & 0.626 & 0.066 & 0.062 & 0.064 & 0.100 & 0.072 \\
\hline X26-Toilets-washrooms & 0.347 & 0.110 & 0.223 & 0.222 & 0.049 & 0.452 & 0.096 & -0.039 & 0.203 & 0.073 & 0.232 \\
\hline X27-F\&B Cost* & -0.094 & 0.245 & 0.061 & 0.025 & 0.024 & -0.026 & 0.722 & 0.043 & -0.095 & -0.049 & -0.103 \\
\hline X28-Transport cost * & -0.050 & 0.020 & 0.265 & 0.102 & 0.077 & -0.062 & 0.716 & -0.145 & -0.093 & 0.003 & -0.065 \\
\hline X29-Accommodation cost* & 0.058 & 0.025 & 0.021 & 0.321 & 0.007 & 0.095 & 0.689 & 0.014 & -0.040 & 0.066 & 0.018 \\
\hline X30-Prices of tours * & 0.067 & 0.257 & 0.002 & 0.002 & 0.090 & -0.229 & 0.634 & -0.088 & 0.136 & -0.070 & -0.070 \\
\hline X31-Handicrafts & 0.021 & 0.111 & 0.116 & 0.233 & 0.003 & -0.045 & 0.058 & 0.748 & -0.029 & 0.020 & 0.159 \\
\hline X32-Costume & 0.057 & 0.310 & 0.010 & 0.086 & 0.081 & 0.081 & -0.048 & 0.670 & 0.019 & 0.119 & -0.002 \\
\hline X33-Monuments & 0.133 & 0.110 & 0.178 & 0.002 & 0.038 & -0.007 & -0.168 & 0.663 & 0.141 & 0.042 & -0.131 \\
\hline X34-Information centers & 0.111 & 0.110 & 0.154 & 0.093 & 0.097 & 0.203 & -0.050 & 0.033 & 0.826 & 0.058 & 0.083 \\
\hline X35-Licensed guides & 0.133 & 0.127 & 0.205 & 0.077 & 0.025 & 0.244 & -0.048 & 0.086 & 0.783 & 0.018 & 0.031 \\
\hline X36-Climatic pleasantness & 0.018 & 0.024 & 0.156 & 0.109 & 0.127 & 0.038 & -0.065 & 0.093 & -0.054 & 0.772 & 0.012 \\
\hline X37-Natural beauty & 0.057 & 0.138 & 0.000 & 0.145 & 0.205 & -0.019 & 0.035 & 0.055 & 0.112 & 0.742 & 0.026 \\
\hline
\end{tabular}




\begin{tabular}{|l|c|c|c|c|c|c|c|c|c|c|c|}
\hline Variables & FAC1 & FAC2 & FAC3 & FAC4 & FAC5 & FAC6 & FAC7 & FAC8 & FAC9 & FAC10 & FAC11 \\
\hline $\begin{array}{l}\text { X38-Sightseeing } \\
\text { opportunities }\end{array}$ & 0.012 & 0.019 & 0.012 & 0.109 & 0.038 & 0.081 & -0.069 & -0.020 & -0.036 & 0.059 & 0.893 \\
\hline X39-Heritage walk & 0.088 & 0.081 & 0.205 & 0.091 & 0.148 & 0.070 & 0.198 & 0.080 & 0.334 & 0.047 & 0.593 \\
\hline Eigen values & 3.114 & 3.113 & 3.105 & 2.719 & 2.703 & 2.438 & 2.222 & 1.866 & 1.849 & 1.417 & 1.397 \\
\hline Variance explained & 24.661 & 6.598 & 6.064 & 5.236 & 4.743 & 4.057 & 3.794 & 3.194 & 2.866 & 2.751 & 2.606 \\
\hline
\end{tabular}

*Inverse coded

The factor loading is suppressed by 0.50 (Cerit, 2000; Hair et al., 2010; Pantouvakis, 2006; Smith, 1987). The loadings suggest that no item is required to delete as loadings of all items were greater than 0.50 . Eleven factors were extracted with Eigen values greater than 1 . In all eleven factors explained 66.5 percent of the variance. The factors of tourism attractiveness are presented in Table 2 with their loadings, Eigen values, and percentage of variance explained.

The eleven factors have been used to prepare the Index of Destination Attractiveness (IDA) based on their weightings presented in Table 3.

Table 3. Factor and variables weightings

\begin{tabular}{|c|c|c|c|c|c|c|c|c|c|c|c|}
\hline Variable & FAC1 & FAC2 & FAC3 & FAC4 & FAC5 & FAC6 & FAC7 & FAC8 & FAC9 & FAC10 & FAC11 \\
\hline $\mathrm{X} 1$ & 0.20 & 0.00 & 0.02 & 0.00 & 0.00 & 0.00 & 0.00 & 0.01 & 0.01 & 0.00 & 0.00 \\
\hline$x 2$ & 0.20 & 0.00 & 0.01 & 0.00 & 0.01 & 0.00 & 0.00 & 0.00 & 0.00 & 0.00 & 0.01 \\
\hline$x 3$ & 0.17 & 0.01 & 0.01 & 0.00 & 0.00 & 0.02 & 0.00 & 0.00 & 0.00 & 0.00 & 0.00 \\
\hline$X 4$ & 0.16 & 0.01 & 0.00 & 0.04 & 0.01 & 0.01 & 0.00 & 0.00 & 0.00 & 0.00 & 0.01 \\
\hline$\times 5$ & 0.00 & 0.21 & 0.00 & 0.01 & 0.01 & 0.00 & 0.00 & 0.00 & 0.00 & 0.00 & 0.00 \\
\hline$x 6$ & 0.00 & 0.18 & 0.01 & 0.02 & 0.00 & 0.02 & 0.01 & 0.00 & 0.00 & 0.00 & 0.00 \\
\hline$x 7$ & 0.00 & 0.17 & 0.00 & 0.00 & 0.00 & 0.00 & 0.00 & 0.06 & 0.00 & 0.00 & 0.00 \\
\hline$x 8$ & 0.01 & 0.12 & 0.02 & 0.02 & 0.01 & 0.00 & 0.00 & 0.01 & 0.01 & 0.01 & 0.00 \\
\hline$\times 9$ & 0.00 & 0.06 & 0.00 & 0.03 & 0.00 & 0.01 & 0.01 & 0.02 & 0.01 & 0.01 & 0.00 \\
\hline $\mathrm{X} 10$ & 0.02 & 0.00 & 0.21 & 0.01 & 0.00 & 0.01 & 0.00 & 0.00 & 0.00 & 0.00 & 0.00 \\
\hline $\mathrm{X} 11$ & 0.02 & 0.00 & 0.16 & 0.00 & 0.00 & 0.02 & 0.00 & 0.01 & 0.01 & 0.00 & 0.00 \\
\hline $\mathrm{X} 12$ & 0.02 & 0.02 & 0.16 & 0.00 & 0.00 & 0.00 & 0.01 & 0.00 & 0.01 & 0.03 & 0.00 \\
\hline $\mathrm{X} 13$ & 0.02 & 0.01 & 0.13 & 0.00 & 0.01 & 0.00 & 0.03 & 0.00 & 0.01 & 0.00 & 0.00 \\
\hline X14 & 0.01 & 0.00 & 0.06 & 0.06 & 0.02 & 0.01 & 0.01 & 0.03 & 0.00 & 0.01 & 0.02 \\
\hline X15 & 0.01 & 0.01 & 0.00 & 0.23 & 0.00 & 0.00 & 0.00 & 0.00 & 0.00 & 0.03 & 0.00 \\
\hline $\mathrm{X} 16$ & 0.03 & 0.02 & 0.00 & 0.20 & 0.00 & 0.00 & 0.01 & 0.00 & 0.00 & 0.00 & 0.00 \\
\hline $\mathrm{X} 17$ & 0.01 & 0.01 & 0.00 & 0.20 & 0.01 & 0.00 & 0.00 & 0.02 & 0.00 & 0.01 & 0.00 \\
\hline $\mathrm{X} 18$ & 0.00 & 0.01 & 0.05 & 0.06 & 0.03 & 0.01 & 0.00 & 0.01 & 0.05 & 0.01 & 0.01 \\
\hline X19 & 0.00 & 0.00 & 0.00 & 0.00 & 0.26 & 0.00 & 0.00 & 0.00 & 0.00 & 0.00 & 0.00 \\
\hline$x 20$ & 0.00 & 0.00 & 0.00 & 0.00 & 0.24 & 0.00 & 0.00 & 0.00 & 0.00 & 0.00 & 0.01 \\
\hline$x 21$ & 0.00 & 0.01 & 0.01 & 0.00 & 0.22 & 0.00 & 0.00 & 0.00 & 0.00 & 0.02 & 0.01 \\
\hline$x 22$ & 0.01 & 0.01 & 0.00 & 0.00 & 0.14 & 0.01 & 0.00 & 0.01 & 0.00 & 0.00 & 0.01 \\
\hline X23 & 0.01 & 0.00 & 0.01 & 0.00 & 0.00 & 0.29 & 0.01 & 0.00 & 0.01 & 0.00 & 0.00 \\
\hline
\end{tabular}




\begin{tabular}{|c|c|c|c|c|c|c|c|c|c|c|c|}
\hline Variable & FAC1 & FAC2 & FAC3 & FAC4 & FAC5 & FAC6 & FAC7 & FAC8 & FAC9 & FAC10 & FAC11 \\
\hline$x 24$ & 0.00 & 0.01 & 0.01 & 0.00 & 0.00 & 0.25 & 0.01 & 0.00 & 0.03 & 0.00 & 0.00 \\
\hline$\times 25$ & 0.03 & 0.01 & 0.01 & 0.01 & 0.00 & 0.16 & 0.00 & 0.00 & 0.00 & 0.01 & 0.00 \\
\hline$x 26$ & 0.04 & 0.00 & 0.02 & 0.02 & 0.00 & 0.08 & 0.00 & 0.00 & 0.02 & 0.00 & 0.04 \\
\hline$\times 27$ & 0.00 & 0.02 & 0.00 & 0.00 & 0.00 & 0.00 & 0.23 & 0.00 & 0.00 & 0.00 & 0.01 \\
\hline X28 & 0.00 & 0.00 & 0.02 & 0.00 & 0.00 & 0.00 & 0.23 & 0.01 & 0.00 & 0.00 & 0.00 \\
\hline$\times 29$ & 0.00 & 0.00 & 0.00 & 0.04 & 0.00 & 0.00 & 0.21 & 0.00 & 0.00 & 0.00 & 0.00 \\
\hline$\times 30$ & 0.00 & 0.02 & 0.00 & 0.00 & 0.00 & 0.02 & 0.18 & 0.00 & 0.01 & 0.00 & 0.00 \\
\hline X31 & 0.00 & 0.00 & 0.00 & 0.02 & 0.00 & 0.00 & 0.00 & 0.30 & 0.00 & 0.00 & 0.02 \\
\hline X32 & 0.00 & 0.03 & 0.00 & 0.00 & 0.00 & 0.00 & 0.00 & 0.24 & 0.00 & 0.01 & 0.00 \\
\hline$\times 33$ & 0.01 & 0.00 & 0.01 & 0.00 & 0.00 & 0.00 & 0.01 & 0.24 & 0.01 & 0.00 & 0.01 \\
\hline X34 & 0.00 & 0.00 & 0.01 & 0.00 & 0.00 & 0.02 & 0.00 & 0.00 & 0.37 & 0.00 & 0.00 \\
\hline$\times 35$ & 0.01 & 0.01 & 0.01 & 0.00 & 0.00 & 0.02 & 0.00 & 0.00 & 0.33 & 0.00 & 0.00 \\
\hline$\times 36$ & 0.00 & 0.00 & 0.01 & 0.00 & 0.01 & 0.00 & 0.00 & 0.00 & 0.00 & 0.42 & 0.00 \\
\hline$\times 37$ & 0.00 & 0.01 & 0.00 & 0.01 & 0.02 & 0.00 & 0.00 & 0.00 & 0.01 & 0.39 & 0.00 \\
\hline$\times 38$ & 0.00 & 0.00 & 0.00 & 0.00 & 0.00 & 0.00 & 0.00 & 0.00 & 0.00 & 0.00 & 0.57 \\
\hline X39 & 0.00 & 0.00 & 0.01 & 0.00 & 0.01 & 0.00 & 0.02 & 0.00 & 0.06 & 0.00 & 0.25 \\
\hline Total & 1.00 & 1.00 & 1.00 & 1.00 & 1.00 & 1.00 & 1.00 & 1.00 & 1.00 & 1.00 & 1.00 \\
\hline Factor weight & 0.12 & 0.12 & 0.12 & 0.10 & 0.10 & 0.09 & 0.09 & 0.07 & 0.07 & 0.05 & 0.05 \\
\hline
\end{tabular}

IDA is based on the assumption that the weights of variables under every factor should be equal to 1 . The unrepresented weights are referred to as residuals. The residual allows the sum of correlated variables to be equal to 1 . The IDA is calculated using the following formula;

$\sum_{i=1}^{n} \alpha_{i} \cdot \bar{X}_{i}$

Where, $\alpha_{i}$ indicates variable weight, $\bar{X}_{i}$ is indicates the mean of the variable. The sum of weights; variables and factors should be equal to 1 (Krešić, Prebezac, 2011).

The weightings presented in Table 3 would be used to define the equations to prepare the attractiveness index value for every single factor and an aggregate attractiveness value for the Kashmir valley. The equations with the name of factors are defined below;

FAC1 (Hygiene and cleanliness)

$=\left(\bar{X}_{1} \cdot 0.20\right)+\left(\bar{X}_{2} \cdot 0.20\right)+\left(\bar{X}_{3} \cdot 0.17\right)+\left(\bar{X}_{4} \cdot 0.16\right)+\left(\bar{X}_{f 1} \cdot 0.27\right)$

FAC2 (Food attractions)

$=\left(\bar{X}_{5} \cdot 0.21\right)+\left(\bar{X}_{6} \cdot 0.18\right)+\left(\bar{X}_{7} \cdot 0.17\right)+\left(\bar{X}_{8} \cdot 0.12\right)+\left(\bar{X}_{9} \cdot 0.06\right)+\left(\bar{X}_{f 2} \cdot 0.26\right)$

$\mathrm{FAC}_{3}$ (Transport facilities)

$=\left(\bar{X}_{10} \cdot 0.21\right)+\left(\bar{X}_{11} \cdot 0.16\right)+\left(\bar{X}_{12} \cdot 0.16\right)+\left(\bar{X}_{13} \cdot 0.13\right)+\left(\bar{X}_{14} \cdot 0.06\right)+\left(\bar{X}_{f 3} \cdot 0.28\right)$ 
$\mathrm{FAC}_{4}$ (Accommodation facilities)

$=\left(\bar{X}_{15} \cdot 0.23\right)+\left(\bar{X}_{16} \cdot 0.20\right)+\left(\bar{X}_{17} \cdot 0.20\right)+\left(\bar{X}_{18} \cdot 0.06\right)+\left(\bar{X}_{f 4} \cdot 0.31\right)$

$\mathrm{FAC}_{5}$ (Site attractions)

$=\left(\bar{X}_{19} \cdot 0.26\right)+\left(\bar{X}_{20} \cdot 0.24\right)+\left(\bar{X}_{21} \cdot 0.22\right)+\left(\bar{X}_{22} \cdot 0.14\right)+\left(\bar{X}_{f 5} \cdot 0.14\right)$

FAC6 (Communication facilities)

$=\left(\bar{X}_{23} \cdot 0.29\right)+\left(\bar{X}_{24} \cdot 0.25\right)+\left(\bar{X}_{25} \cdot 0.16\right)+\left(\bar{X}_{26} \cdot 0.08\right)+\left(\bar{X}_{f 6} \cdot 0.22\right)$

$\mathrm{FAC}_{7}$ (Cost)

$=\left(\bar{X}_{27} \cdot 0.39\right)+\left(\bar{X}_{28} \cdot 0.23\right)+\left(\bar{X}_{29} \cdot 0.21\right)+\left(\bar{X}_{30} \cdot 0.18\right)+\left(\bar{X}_{f 7} \cdot 0.15\right)$

FAC8 (Cultural attractions)

$=\left(\bar{X}_{31} \cdot 0.30\right)+\left(\bar{X}_{32} \cdot 0.24\right)+\left(\bar{X}_{33} \cdot 0.24\right)+\left(\bar{X}_{f 8} \cdot 0.22\right)$

FAC9 (Tourist amenities)

$=\left(\bar{X}_{34} \cdot 0.37\right)+\left(\bar{X}_{35} \cdot 0.33\right)+\left(\bar{X}_{f 9} \cdot 0.30\right)$

FAC10 (Natural attractions)

$=\left(\bar{X}_{36} \cdot 0.42\right)+\left(\bar{X}_{37} \cdot 0.39\right)+\left(\bar{X}_{f 10} \cdot 0.19\right)$

FAC11 (Tourist activity)

$=\left(\bar{X}_{38} \cdot 0.57\right)+\left(\bar{X}_{39} \cdot 0.25\right)+\left(\bar{X}_{f 11} \cdot 0.18\right)$

\section{IDA (Aggregate index destination attractiveness)}

$=\left(\mathrm{FAC}_{1} \cdot 0.12\right)+\left(\mathrm{FAC}_{2} \cdot 0.12\right)+\left(\mathrm{FAC}_{3} \cdot 0.12\right)+\left(\mathrm{FAC}_{4} \cdot 0.10\right)+\left(\mathrm{FAC}_{5} \cdot 10\right)+\left(\mathrm{FAC}_{6} \cdot 0.09\right)+\left(\mathrm{FAC}_{7} \cdot 0.09\right)+$ $+\left(\mathrm{FAC}_{8} \cdot 0.07\right)+\left(\mathrm{FAC}_{9} \cdot 0.07\right)+\left(\mathrm{FAC}_{10} \cdot 0.05\right)+\left(\mathrm{FAC}_{11} \cdot 0.05\right)$

\section{Index of Destination Attractiveness of Kashmir valley}

The attractiveness index model for Kashmir valley is presented in Table 4. The aggregated IDA value of valley (3.363) is taken as a benchmark to judge the attractiveness of different factors. 
Table 4. Attractiveness index for Kashmir

\begin{tabular}{|c|c|c|c|c|c|}
\hline \multirow{2}{*}{\multicolumn{2}{|c|}{ Factors and variables }} & \multicolumn{2}{|c|}{ Loadings } & \multirow{3}{*}{$\begin{array}{c}\text { Mean } \\
5 \\
\end{array}$} & \multirow{3}{*}{$\begin{array}{c}\text { IDA values } \\
6\end{array}$} \\
\hline & & \multirow{2}{*}{$\begin{array}{c}\text { Variable } \\
3\end{array}$} & \multirow{2}{*}{$\begin{array}{c}\text { Factor } \\
4 \\
\end{array}$} & & \\
\hline 1 & 2 & & & & \\
\hline $\begin{array}{l}\text { FAC1 } \\
\text { X1 } \\
\text { X2 } \\
\text { X3 } \\
\text { X4 } \\
\text { F1 }\end{array}$ & $\begin{array}{l}\text { Hygiene and cleanliness } \\
\text { At transportation } \\
\text { At sites } \\
\text { At food outlets } \\
\text { At accommodation } \\
\text { Residual }\end{array}$ & $\begin{array}{l}0.20 \\
0.20 \\
0.17 \\
0.16 \\
0.27\end{array}$ & 0.12 & $\begin{array}{l}3.316 \\
3.397 \\
3.268 \\
3.662 \\
3.411\end{array}$ & $\begin{array}{l}3.405 \\
0.663 \\
0.679 \\
0.556 \\
0.586 \\
0.921\end{array}$ \\
\hline $\begin{array}{l}\text { FAC2 } \\
X 5 \\
\text { X6 } \\
\text { X7 } \\
\text { X8 } \\
\text { X9 } \\
\text { F2 }\end{array}$ & $\begin{array}{l}\text { Food attractions } \\
\text { Food quality } \\
\text { Food varieties } \\
\text { Local food } \\
\text { Customer support } \\
\text { Hospitality } \\
\text { Residual }\end{array}$ & $\begin{array}{l}0.21 \\
0.18 \\
0.17 \\
0.12 \\
0.06 \\
0.26\end{array}$ & 0.12 & $\begin{array}{l}3.538 \\
3.362 \\
3.554 \\
3.646 \\
4.043 \\
3.629 \\
\end{array}$ & $\begin{array}{l}3.576 \\
0.743 \\
0.605 \\
0.604 \\
0.438 \\
0.243 \\
0.944 \\
\end{array}$ \\
\hline $\begin{array}{l}\mathrm{FAC3} \\
\mathrm{X} 10 \\
\mathrm{X} 11 \\
\mathrm{X} 12 \\
\mathrm{X} 13 \\
\mathrm{X} 14 \\
\mathrm{~F} 3\end{array}$ & $\begin{array}{l}\text { Transport facilities } \\
\text { Inside transport } \\
\text { Accessibility over world } \\
\text { Quality of transport } \\
\text { Road signage } \\
\text { Shopping } \\
\text { Residual }\end{array}$ & $\begin{array}{l}0.21 \\
0.16 \\
0.16 \\
0.13 \\
0.06 \\
0.28\end{array}$ & 0.12 & $\begin{array}{l}3.327 \\
3.243 \\
3.372 \\
3.268 \\
3.418 \\
3.326\end{array}$ & $\begin{array}{l}3.318 \\
0.699 \\
0.519 \\
0.540 \\
0.425 \\
0.205 \\
0.931\end{array}$ \\
\hline $\begin{array}{l}\text { FAC4 } \\
\times 15 \\
\times 16 \\
\times 17 \\
\times 18 \\
\text { F4 }\end{array}$ & $\begin{array}{l}\text { Accommodation facilities } \\
\text { Accommodation varieties } \\
\text { Accommodation quality } \\
\text { Accommodation location } \\
\text { Recreational activities } \\
\text { Residual }\end{array}$ & $\begin{array}{l}0.23 \\
0.20 \\
0.20 \\
0.06 \\
0.31\end{array}$ & 0.10 & $\begin{array}{l}3.811 \\
3.756 \\
3.970 \\
3.584 \\
3.780\end{array}$ & $\begin{array}{l}3.809 \\
0.877 \\
0.751 \\
0.794 \\
0.215 \\
1.172\end{array}$ \\
\hline $\begin{array}{l}\text { FAC5 } \\
\times 19 \\
\times 20 \\
\times 21 \\
\times 22 \\
\text { F5 }\end{array}$ & $\begin{array}{l}\text { Site attraction } \\
\text { Pahalgam } \\
\text { Sonmarg } \\
\text { Gulmarg } \\
\text { Srinagar } \\
\text { Residual }\end{array}$ & $\begin{array}{l}0.26 \\
0.24 \\
0.22 \\
0.14 \\
0.14\end{array}$ & 0.10 & $\begin{array}{l}3.970 \\
3.900 \\
4.235 \\
3.916 \\
4.005\end{array}$ & $\begin{array}{l}4.009 \\
1.032 \\
0.936 \\
0.932 \\
0.548 \\
0.561\end{array}$ \\
\hline $\begin{array}{l}\text { FAC6 } \\
\times 23 \\
\times 24 \\
\times 25 \\
\times 26 \\
\text { F7 }\end{array}$ & $\begin{array}{l}\text { Communication facilities } \\
\text { Cellular services } \\
\text { Internet services } \\
\text { Banking and ATMs } \\
\text { Toilets and washrooms } \\
\text { Residual }\end{array}$ & $\begin{array}{l}0.29 \\
0.25 \\
0.16 \\
0.08 \\
0.22\end{array}$ & 0.09 & $\begin{array}{l}2.465 \\
2.535 \\
3.046 \\
2.962 \\
2.752\end{array}$ & $\begin{array}{l}2.687 \\
0.715 \\
0.634 \\
0.487 \\
0.237 \\
0.605\end{array}$ \\
\hline $\begin{array}{l}\mathrm{FAC7} \\
\times 27 \\
\times 28 \\
\times 29 \\
\times 30 \\
\mathrm{~F} 7\end{array}$ & $\begin{array}{l}\text { Cost } \\
\text { Food and beverage cost } \\
\text { Transport cost } \\
\text { Accommodation cost } \\
\text { Prices of tours } \\
\text { Residual }\end{array}$ & $\begin{array}{l}0.23 \\
0.23 \\
0.21 \\
0.18 \\
0.15\end{array}$ & 0.09 & $\begin{array}{l}2.659 \\
2.749 \\
2.597 \\
2.803 \\
2.702 \\
\end{array}$ & $\begin{array}{l}2.699 \\
0.612 \\
0.632 \\
0.545 \\
0.505 \\
0.405\end{array}$ \\
\hline $\begin{array}{l}\text { FAC8 } \\
\times 31 \\
\times 32 \\
\times 33 \\
\text { F8 }\end{array}$ & $\begin{array}{l}\text { Cultural attractions } \\
\text { Handicrafts } \\
\text { Costume } \\
\text { Monuments } \\
\text { Residual }\end{array}$ & $\begin{array}{l}0.30 \\
0.24 \\
0.24 \\
0.22\end{array}$ & 0.07 & $\begin{array}{l}3.803 \\
3.565 \\
3.649 \\
3.672\end{array}$ & $\begin{array}{c}3.680 \\
1.141 \\
0.856 \\
0.876 \\
0.808\end{array}$ \\
\hline
\end{tabular}




\begin{tabular}{|c|c|c|c|c|c|}
\hline \multirow{2}{*}{\multicolumn{2}{|c|}{ Factors and variables }} & \multicolumn{2}{|c|}{ Loadings } & \multirow{3}{*}{$\begin{array}{c}\text { Mean } \\
5\end{array}$} & \multirow{3}{*}{$\begin{array}{c}\text { IDA values } \\
6\end{array}$} \\
\hline & & \multirow{2}{*}{$\begin{array}{c}\text { Variable } \\
3\end{array}$} & \multirow{2}{*}{$\begin{array}{c}\text { Factor } \\
4\end{array}$} & & \\
\hline 1 & 2 & & & & \\
\hline $\begin{array}{l}\text { FAC9 } \\
\times 34 \\
\times 35 \\
\text { F9 }\end{array}$ & $\begin{array}{l}\text { Tourist amenities } \\
\text { Information centers } \\
\text { Licensed guides } \\
\text { Residual }\end{array}$ & $\begin{array}{l}0.37 \\
0.33 \\
0.30\end{array}$ & 0.07 & $\begin{array}{l}3.016 \\
2.978 \\
2.997\end{array}$ & $\begin{array}{l}2.998 \\
1.116 \\
0.983 \\
0.899\end{array}$ \\
\hline $\begin{array}{l}\text { FAC10 } \\
\times 36 \\
\times 37 \\
\text { F10 }\end{array}$ & $\begin{array}{l}\text { Natural attractions } \\
\text { Climatic pleasantness } \\
\text { Natural beauty } \\
\text { Residual }\end{array}$ & $\begin{array}{l}0.42 \\
0.39 \\
0.19\end{array}$ & 0.05 & $\begin{array}{l}4.378 \\
4.768 \\
4.537\end{array}$ & $\begin{array}{l}4.560 \\
1.839 \\
1.860 \\
0.862\end{array}$ \\
\hline $\begin{array}{l}\text { FAC11 } \\
\text { X38 } \\
\text { X39 } \\
\text { F11 }\end{array}$ & $\begin{array}{l}\text { Tourist activity } \\
\text { Sightseeing opportunities } \\
\text { Heritage walk } \\
\text { Residual }\end{array}$ & $\begin{array}{l}0.57 \\
0.25 \\
0.18\end{array}$ & 0.05 & $\begin{array}{l}3.289 \\
3.362 \\
3.326\end{array}$ & $\begin{array}{l}3.314 \\
1.875 \\
0.841 \\
0.599\end{array}$ \\
\hline \multicolumn{5}{|c|}{ Aggregated value for index destination attractiveness (IDA) } & 3.363 \\
\hline
\end{tabular}

The index values suggest natural attractions and site attraction are rated high in terms of attractiveness. The IDA values for both factors are 4.560 and 4.009 respectively. Further, IDA value for factors such as accommodation facilities, cultural attractions, and food attractions is $3.809,3.608$, and 3.576 respectively. These factors are followed by hygiene and cleanliness (3.405) transport facilities (3.318) and tourists' activity (3.314). The IDA values were found low for tourist amenities (2.998), Cost (2.699), and communication facilities (2.687).

The identified factors and their IDA values have been used to know if these can be categorized as Motivators and Hygiene factors based on Herzberg classification as has been done in earlier studies (Crompton, 2003; Jensen, 2007; Tkaczynski, Rundle, 2013). The motivators have been identified based on earlier studies of touristic attractiveness and motivation (see Table 5).

Table 5. Classification of factors

\begin{tabular}{|c|l|c|c|l|}
\hline Factor & Factors Name & IDA Value & Classification & Studies using motivators and hygiene \\
\hline FAC10 & Natural attractions & 4.560 & Motivator & Baloglu, Usyal, (1996); Jensen, (2007) \\
\hline FAC5 & Site attractions & 4.009 & Motivator & Lim et.al. (2015); Jensen, (2007); Sharma, (2016) \\
\hline FAC4 & Accommodation facilities & 3.809 & Hygiene & $\begin{array}{l}\text { Crompton, (2003); Jensen, (2007); Vengesayi et.al. } \\
\text { (2009) }\end{array}$ \\
\hline FAC8 & Cultural attractions & 3.680 & Motivator & Chaudhary, (2000); Cromption, (2003); Jensen, (2007) \\
\hline FAC2 & Food attractions & 3.576 & Motivator & Jensen, (2007); Quan, Wang, (2003) \\
\hline FAC1 & Hygiene and Cleanliness & 3.405 & Hygiene & Chaudhary, (2000); Jensen, (2007) \\
\hline FAC3 & Transport facilities & 3.318 & Hygiene & Jensen, (2007); Vengesayi et al. (2009) \\
\hline FAC11 & Tourist activity & 3.314 & Motivator & $\begin{array}{l}\text { Crompton, (2003); Jensen, (2007); Tkaczynski, Rundle, } \\
\text { (2013) }\end{array}$ \\
\hline FAC9 & Tourist amenities & 2.988 & Hygiene & Barker, Crompton, (2000); Jensen, (2007) \\
\hline FAC7 & Cost & 2.699 & Motivator & Lou, (2014); Yuan, McDonald, (1990) \\
\hline FAC6 & Communication facilities & 2.687 & Hygiene & Jensen, (2007); Vengesayi et.al. (2009) \\
\hline
\end{tabular}

The classification of factors showed six factors are having IDA value above aggregated value and five factors have lesser values. These have been placed in the matrix. 
Table 6. Attractiveness matrix

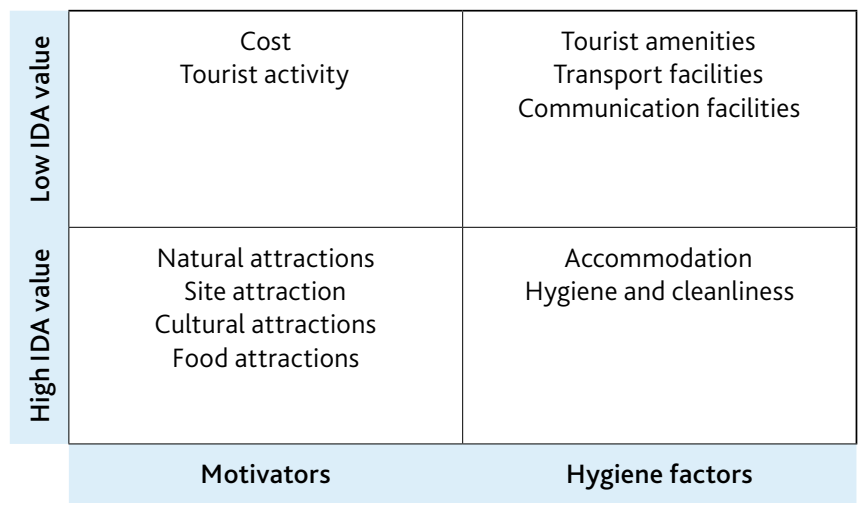

The matrix Table 6 suggests that most of the motivating factors are rated high except cost and tourist activity. The natural and site attractions are rated high endorsing the common perception about Kashmir valley. However, food as an attraction is also rated high. The valley lacks on most of tourist amenities and facilities (hygiene factors) except accommodation and cleanliness.

\section{Conclusions}

The purpose of this study was to study tourism attractiveness of Kashmir valley in India and also to develop a measure that can evolve as a standard but evolving Index. An Index of Destination Attractiveness (IDA) has been developed and used to measure tourism attractiveness of Kashmir valley in India. The IDA helps in identifying eleven factors perceived important for attractiveness by tourists and are labeled as hygiene and cleanliness, food attractions, transport facilities, accommodation facilities, site attractiveness, communication facilities, cost, cultural attractions, tourist amenities, natural attractions, and tourist activity. Each factor further has a number of variables specific to valley. While these findings can be useful for destination managers to work on deficient areas and improve overall attractiveness, the index developed can be used annually or at any other appropriate interval for a sustained destination management programme. The repeat use of IDA will also help in fine tuning this index further and incorporate more factors as warranted by the future shape of destination Kashmir valley. IDA prepared for this study can also find application at other destinations with little modifications as warranted by the features of attractiveness these destinations.

\section{References}

An, L.T., Markowski, J., Bartos, M., Rzenca, A., Namiecinski, P. 2019. An evaluation of destination attractiveness for nature-based tourism: Recommendations for the management of national parks in Vietnam. Nature Conservation 32, 51-80.

Baker, D. A., Crompton, J. L. 200o. Quality, satisfaction and behavioral intentions. Annals of tourism research 27(3), 785-804.

Baloglu, S., Uysal, M. 1996. Market segments of push and pull motivations: A canonical correlation approach. International Journal of Contemporary Hospitality Management 8(3), $32-38$. 
Bhat, I. A., PJ, S. 2015. Pilgrimage Tourism in Sindh Valley (Kashmir): A Case Study of Kheer Bhawani Shrine. Atna Journal of Tourism Studies 9 (1), 36-61.

Bhat, M. A., Qadir, N. 2013. Tourist satisfaction in Kashmir: An empirical assessment. Journal of Business Theory and Practice 1(1), 152-166.

Blazeska, D., Milenkovski, A., Gramatnikovski, S. 2015. The quality of the tourist destinations a key factor for increasing their attractiveness. UTMS Journal of Economics 6 (2), 341-353.

Castro, E. V., Souza, T. B., Thapa, B. 2015. Determinants of tourism attractiveness in the national parks of Brazil. Parks 21(2), 51-62.

Cerit, A. G. 200o. Maritime transport as an area of competitive advantage in international marketing. International journal of maritime economics 2(1), 49-67.

Chaudhary, M. 200o. India's image as a tourist destination-a perspective of foreign tourists. Tourism management 21(3), 293-297.

Chaudhary, M., Rani P., Sheokand R. 2017. Attractiveness of Bhagavat Gita and its symbols among tourists visiting Kurukshetra. Indian journal of International Tourism E Hospitality Research 8(1), 1-18.

Chaudhary, M. (2010). Tourism marketing. Oxford University Press: New Delhi.

Chaudhary, M., Islam, N.U. 2020. Identification and Mapping of Religious Tourist Resources in Kashmir Valley. International Journal of Hospitality \& Tourism Systems 13(1), 13-21.

Cho, V. 2008. Linking location attractiveness and tourist intention. Tourism and Hospitality Research 8(3), 220-224.

Cracolici, M. F., Nijkamp, P. 2009. The attractiveness and competitiveness of tourist destinations: A study of Southern Italian regions. Tourism management 3o(3), 336-344.

Crompton, J. L. 2003. Adapting Herzberg: a conceptualization of the effects of hygiene and motivator attributes on perceptions of event quality. Journal of travel research 41, 305-310.

Cugno, M., Grimmer, M., Viassone, M. 2012. Measuring local tourism attractiveness: The case of Italy. ANZAM 2012. Proceedings 26th Australian E New Zealand Academy of Management (ANZAM) Conference (pp. 1-22).

Das, D., Sharma, S. K., Mohapatra, P. K., Sarkar, A. 2007. Factors influencing the attractiveness of a tourist destination: A case study. Journal of Services Research 7(1).

Dupeyras, A., MacCallum, N. 2013. Indicators for measuring competitiveness in tourism: a guidance document. OECD Tourism Papers, 2013/o2, OECD Publishing. Retrieved from http://dx.doi.org/10.1787/5k47t9q2tg23-en

Edward, M., George, B. P. 2008. Tourism development in the State of Kerala, India: A study of destination attractiveness. European journal of tourism research 1(1).

Fakeye, P. C., Crompton, J. L. 1991. Image differences between prospective, first-time, and repeat visitors to the Lower Rio Grande Valley. Journal of travel research 30(2), 10-16.

Formica, S., Uysal, M. 2006. Destination attractiveness based on supply and demand evaluations: An analytical framework. Journal of Travel Research 44(4), 418-430.

Ganie, S. A., Dar, M. A, 2020. Role of Human Resources in the Tourism Industry with Special Reference to Jammu and Kashmir. Turizam 24(2), 67-78

Gearing, C. E., Swart, W. W., Var, T. 1974. Establishing a measure of touristic attractiveness. Journal of travel Research 12(4), 1-8.

Hong-bumm, K. 1998. Perceived attractiveness of Korean destinations. Annals of tourism research 25(2), 340-361.

$\mathrm{Hu}$, Y., Ritchie, J. B. 1993. Measuring destination attractiveness: A contextual approach. Journal of travel research 32(2), 25-34. 
IBEF (2017). Jammu and Kashmir Paradise on earth. Retrieved from https://www.ibef.org/ download/Jammu-Kashmir-January-2017.pdf(Accessed on 20.04.2020)

Islam, S., Hossain, M. K., Noor, M. E. 2017. Determining drivers of destination attractiveness: the case of nature-based tourism of Bangladesh. International Journal of Marketing Studies 9 (3), 10-23.

Jensen, J.M. 2007. An empirical investigation of the relationships between hygiene factors, motivators, satisfaction, and response among visitors to zoos and aquaria. Tourism Review International 11, 307-316

Johnson, C. L. (2017). Millennials' perception of destination attractiveness. (Master's thesis). Retrieved from https://pdfs.semanticscholar.org/oa83/403279002eodc9ce34266do21bo6f 4 bd 4.03f.pdf (Accessed on 05.09.2019)

Joseph, E.U., Udoh, I. S. 2018. Festivities and the tourism destination attractiveness of Akwa Ibom state, Nigeria. International Journal Recent Scientific Research 9(6), 27314-27319.

Kaur, J. 1981. Methodological approach to scenic resource assessment. Tourism Recreation Research 6(1), 19-22.

Kothari C.R. Garg G. (2014). Research Methodology Methods and Techniques. New Age International Publishers, New Delhi, India.

Krešić, D., Prebežac, D. 2011. Index of destination attractiveness as a tool for destination attractiveness assessment. Tourism 59(4), 497-517.

Kumar, R. B., Hafizullah, D. 2017. What makes domestic tourists satisfied: an empirical study of Srinagar (Jammu \& Kashmir). International Journal of Tourism and Travel 10(1), 47-56.

Lawrence, W. R. (1895). The valley of Kashmir. Oxford University press, London.

Laws, E. (1995). Tourist destination management: issues, analysis and policies. Routledge.

Lim, Y. J., Kim, H. K., Lee, T. J. 2016. Visitor motivational factors and level of satisfaction in wellness tourism: Comparison between first-time visitors and repeat visitors. Asia pacific journal of tourism research 21(2), 137-156.

Lou, J. (2014). Analysis of adventure tourist motivation: The case of China-born international students in New Zealand universities (Doctoral dissertation). Retrieved from https:// openrepository.aut.ac.nz/handle/10292/7917(Accessed on 15.09.2019)

Malik, M. I., Bhat, M. S. 2015. Sustainability of tourism development in Kashmir-Is paradise lost? Tourism management perspectives 16, 11-21.

Mayo, E. J., Jarvis, L. P. (1981). The psychology of leisure travel. Effective marketing and selling of travel services. CBI Publishing Company, Inc.

Morachat, C. (2003). A study of destination attractiveness through tourists' perspectives: a focus on Chiang Mai, Thailand. (Doctorial dissertation). Retrieved from https://ro.ecu. edu.au/theses/1504/(Accessed on 10.09.2019)

Mukhopadhyay, D. (2011). Constructing destination attractiveness index - a conceptual note. Retrieved from https://driptoonsustainabletourism.wordpress.com/2011/o8/17/constructing-destination-attractiveness-index-\%E2\%80\%93-a-conceptual-note/(Accessed on 15.06.2019)

Pantouvakis, A. 2006. Port-service quality dimensions and passenger profiles: an exploratory examination and analysis. Maritime Economics $\mathcal{E}$ Logistics 8(4), 402-418.

Paul, S. 2017. Analysing tourism attractiveness using probabilistic travel model: A study of Gangtok and its surroundings. Geografia-Malaysian Journal of Society and Space 9(3), 61-68.

Pompurová, K., Šimočková, I. 2014. Destination attractiveness of Slovakia: perspectives of demand from major tourism source markets. Economics and Management 17 (3), 62-72. 
Quan, S., Wang, N. 2004. Towards a structural model of the tourist experience: An illustration from food experiences in tourism. Tourism management 25(3), 297-305.

Reitsamer, B. F., Brunner-Sperdin, A., Stokburger-Sauer, N. E. 2016. Destination attractiveness and destination attachment: The mediating role of tourists' attitude. Tourism Management Perspectives 19, 93-101.

Ritchie, J. B., Zins, M. 1978. Culture as determinant of the attractiveness of a tourism region. Annals of Tourism Research 5(2), 252-267.

Sharma, S. (2016) India's image as a tourist destination: A study of Incredible India marketing campaign (Unpublished doctorial dissertation), Kurukshetra University, Haryana, India.

Smith, S. L. 1987. Regional analysis of tourism resources. Annals of tourism research 14(2), 254273.

Tkaczynski, A., Rundle-Thiele, S. 2013. Understanding what really motivates attendance: A music festival segmentation study. Journal of Travel $\mathcal{E}$ Tourism Marketing 30(6), 610-623.

Vengesayi, S., Mavondo, F. T., Reisinger, Y. 2009. Tourism destination attractiveness: attractions, facilities, and people as predictors. Tourism Analysis 14(5), 621-636.

Yuan, S., McDonald, C. 1990. Motivational determinates of international pleasure time. Journal of Travel Research 29(1), 42-44.

Zhou, L. (2005). Destination attributes that attract international tourists to Cape Town. (Doctoral dissertation). Retrieved from https:/etd.uwc.ac.za/xmlui/handle/11394/183 (Accessed on 20.09.2019) 